\section{Contextual effects in the discrimination of stop consonant and semivowel}

\section{JOANNE L. MILLER \\ Northeastern University, Boston, Massachusetts 02115}

It has been known for some time that one of the major sources of information for the distinction between a stop consonant (e.g., /b/) and a semivowel (e.g., /w/) in a syllable-initial position is the duration of the initial formant transitions, with shorter transitions specifying a stop consonant and longer transitions specifying a semivowel (Liberman, Delattre, Gerstman, \& Cooper, 1956). Recently, Miller and Liberman (1979) showed that the location of a phonetic boundary along a /ba-wa/ series, whose stimuli varied in transition duration, systematically differed as a function of the overall duration of the syllable. When syllable duration was increased by extending the steady-state formants, a longer transition was required before the percept changed from /ba/ to /wa/. Thus, information occurring later in time-the duration of the steady-state segmentaffected the processing of earlier occurring information-the duration of the initial transitions. Miller and Liberman suggested that the boundary shift may have reflected the operation of mechanisms that compensate for changes in speaking rate; in particular, that the syllable duration provides information about rate and that the listener uses this information when judging a particular transition duration as short or long in order to specify a stop or semivowel, respectively.

The purpose of the present study was to determine whether the influence of syllable duration on the perception of the $/ b /-/ w /$ distinction is reflected in discrimination performance as well as identification performance. Previous studies have shown that when the location of a phonetic boundary along a stimulus series is altered through experimental manipulation such as selective adaptation, there is a shift in the discrimination function that parallels the shift in identification; that is to say, the peaks in the discrimination function before and after adaptation correspond to the phonetic boundary locations before and after adaptation (e.g., Cooper, 1974; Eimas \& Corbit, 1973). The critical issue of concern is whether this is also true when the boundary shift is due not to a temporary alteration in the operating characteristics

This research was supported by NIH Grant NS 14394 to the author, HEW BRS Grant RR 07143 to Northeaste-n Univarsity, and NIH Contract NIH 71-2420 to Haskins Laboratories. I would like to thank Dr. A. M. Liberman for making available the facilities of Haskins Laboratories for the preparation of the stimulus tapes. of the processing system, as in the case of adaptation, but to the inherent acoustic properties of the stimuli being judged. There is at least one study in the literature that has addressed just this issue. Lisker, Liberman, Erickson, Dechovitz, and Mandler (1977), examining the perception of a voicing distinction along a /da-ta/ series, found that the phonetic boundary measured in milliseconds' voice-onset time systematically differed as a function of transition duration and, moreover, that this shift in identification was reflected in discrimination performance. However, note that for the /ba-wa/ syllables, unlike those in the Lisker et al. study, the contextual information (duration of the steady-state formants) is physically distinct from, and occurs after, the information altered to cue the phonetic distinction (the initial formant transitions). It is conceivable that in a discrimination task with these syllables, the listener might respond only on the basis of the critical transitional information being altered, ignoring information about overall duration.

In an attempt to address this issue, identification and discrimination tapes were prepared using two series from the original Miller and Liberman (1979) study: the /ba-wa/ series with an overall syllable duration of $80 \mathrm{msec}$ and the /ba-wa/ series with an overall syllable duration of $296 \mathrm{msec}$. Detailed stimulus specifications are available in the original report. Briefly, each series consisted of 13 syllables that differed from each other in initial transition duration, varying in $4-\mathrm{msec}$ steps from 16 to $64 \mathrm{msec}$. The syllables in the two series differed from each other only in overall syllable duration, 80 or $296 \mathrm{msec}$. Separate identification and discrimination tapes were prepared for each of the two series. The identification tapes contained randomizations of numerous tokens of the 13 stimuli in the series. The discrimination tapes, designed for an oddity task, contained randomizations of all possible triads formed by pairing each syllable in the series with the syllables differing from it by $16 \mathrm{msec}$ of transition duration. Eight subjects were presented the tapes for both the 80 - and the 296-msec series in counterbalanced order. During the identification task, which was presented first, the listeners were asked to identify each syllable as /ba/ or /wa/. During the discrimination task, subjects were told to indicate which of the three syllables in each triad was the odd one. They were further instructed that in many cases the difference between syllables would be very slight-subtle variations in /ba/ and / wa/ - and that they should use any detectable difference in the sounds for making their decision.

Group identification and discrimination performance is displayed in Figure 1. In addition, the individual functions for two representative listeners are 

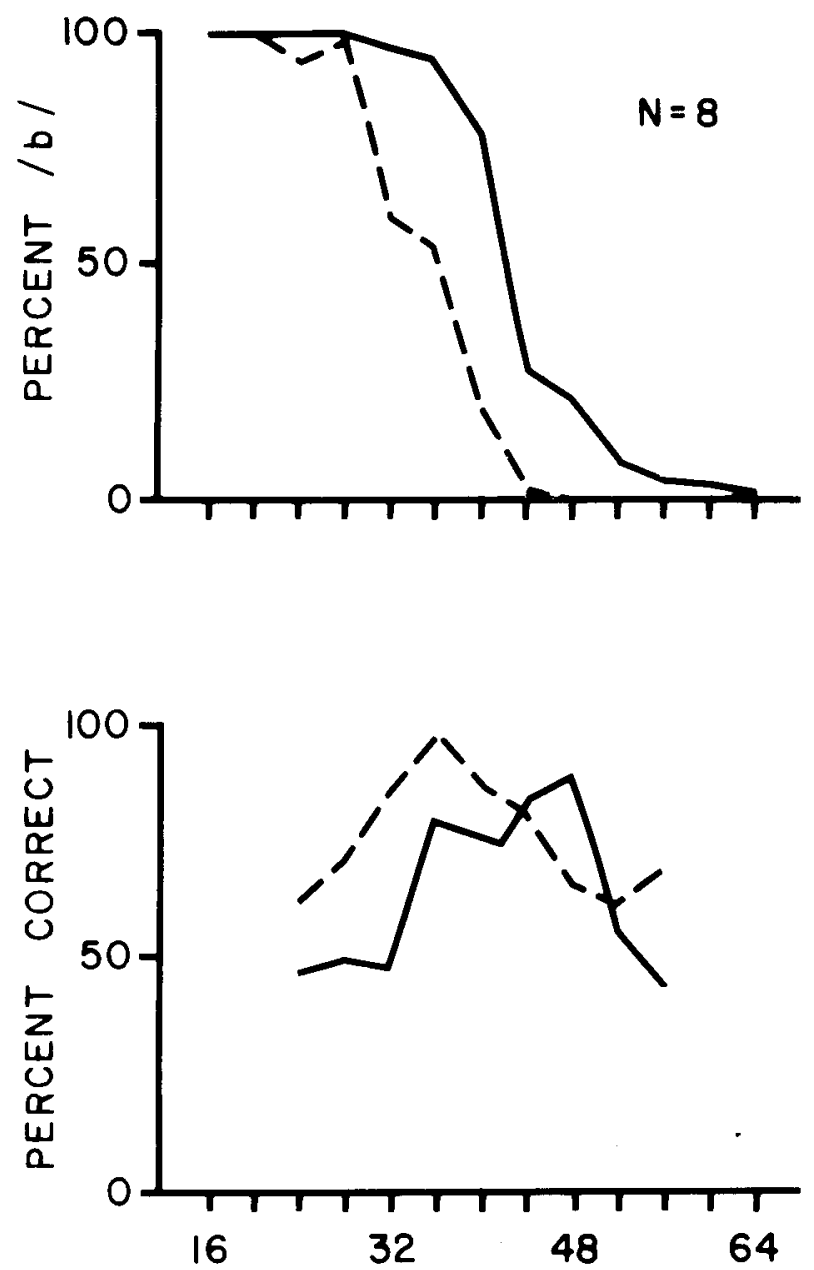

TRANS. DURATION (msec)

Figure 1. Group identification and discrimination performance on the 80-msec series (dashed lines) and the 296-msec series (solid lines) for the eight listeners. Each data point on the identification graph is based on 160 responses ( 20 per subject) and each point on the discrimination graph is based on 144 responses (18 per subject). The discrimination data points are plotted at the transition duration midway between the transition durations of the two stimuli being judged.

displayed in Figure 2. Consider identification performance first. It is apparent from the functions that the listeners yielded the expected shift in labeling with a change in syllable duration: for the syllables with a longer overall duration, the crossover between predominately /b/ responses and predominately /w/ responses was at a longer transition duration. To obtain a measure of the shift, the phonetic boundary value, in milliseconds' transition, was calculated on each series for each subject using a least mean squares analysis. For every subject, the boundary for the 296msec series was at a longer transition duration than that for the $80-\mathrm{msec}$ series $(\mathrm{t}=5.20, \mathrm{p}<.001)$, with a mean shift of $8 \mathrm{msec}$.

Next, turn to a consideration of discrimination performance. The functions indicate that discrimina- tion, like identification, was systematically affected by syllable duration, such that, for a given series, the region of highest discriminability was in the vicinity of the /b/-/w/ boundary. The shift in discrimination as a function of syllable duration was measured in the following way. The location of peak discrimination performance for each subject, on each series, was taken to be the midvalue of the stimulus pair, in milliseconds' transition duration, that yielded the highest discrimination score. For those cases in which maximal performance occurred on more than one pair, the average of the midvalues was considered as the location of the discrimination peak. A comparison of peak locations on the two series indicated that the peak occurred at a longer transition duration on the 296-msec series than on the 80-msec series for seven of the eight listeners $(t=2.59, p<.025)$. Moreover, for the seven listeners who showed a discrimination shift in the predicted direction, there was a correlation of $.80(p<.025)$ between the magnitude of shift in the phonetic boundary and the magnitude of shift in the discrimination peak.

The data show that the ability to discriminate syllables from a /ba-wa/ series that vary in transition duration depends not only on the absolute durations of the transitions, but also on the overall duration of the syllables. Moreover, the close match between identification and discrimination performance suggests that the contextual information is used by the processing system in the same way, regardless of whether the task involves phonetic labeling or discrimination (see Repp, Healy, \& Crowder, 1979, for a recent discussion of the relation between the two tasks). Indeed, it may be the case that the listener is unable to selectively attend to the transition information independently of the steady-state formants and that the processing system necessarily considers the
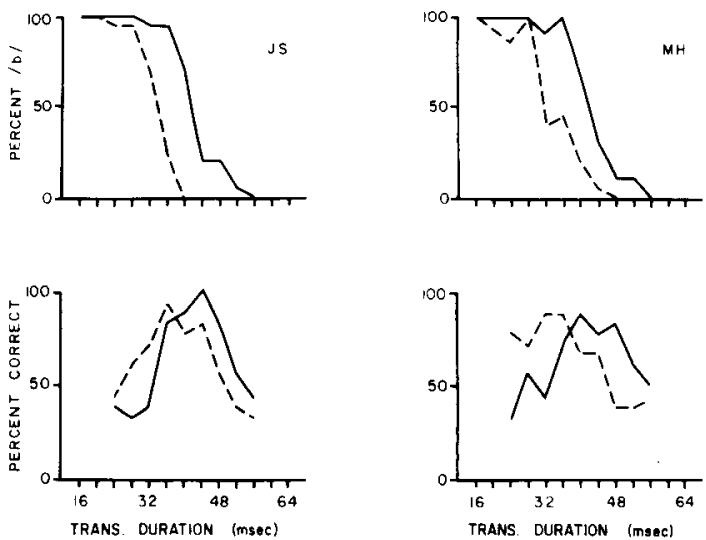

Figure 2. Identification performance on the 80 -msec series (dashed lines) and the 296-msec series (solid lines) for two individual listeners. Each data point on the identification graph is based on 20 responses, and each point on the discrimination graph is based on 18 responses. The discrimination data points are plotted at the transition duration midway between the transition durations of the two stimuli being judged. 
pattern of information across the entire syllable (cf. Mermelstein, 1978). Finally, the present results have important methodological implications. Specifically, the finding that contextual effects of the nature studied are reflected in discrimination data suggests that it may be possible to examine such effects in subject populations for which identification procedures are somewhat problematic, for example, prearticulate infants.

\section{REFERENCES}

Cooper, W. E. Adaptation of phonetic feature analyzers for place of articulation. Journal of the Acoustical Society of America, $1974,56,617-627$.

Eımas, P. D., \& Conbit, J. D. Selective adaptation of linguistic feature detectors. Cognitive Psychology, 1973, 4, 99-109.
Mermelstein, P. On the relationship between vowel and consonant identification when cued by the same acoustic information. Perception \& Psychophysics, 1978, 23, 331-336.

Miller, J. L., \& Liberman, A. M. Some effects of lateroccurring information on the perception of stop consonant and semivowel. Perception \& Psychophysics, 1979, 25, 457-465.

Liberman, A. M., Delattre, P. C., Gerstman, L. J., \& Cooper, F. S. Tempo of frequency change as a cue for distinguishing classes of speech sounds. Journal of Experimental Psychology, 1956, 52, 127-137.

Lisker, L., Liberman, A. M., Erickson, D. M., Dechovitz, D., $\&$ MANDLER, R. On pushing the voice-onset-time (VOT) boundary about. Language and Speech, 1977, 20, 209-216.

RePp, B. H., Healy, A. F., \& Crowder, R. G. Categories and context in the perception of isolated steady-state vowels. Journat of Experimental Psychology: Human Perception and Performance, 1979, 5, 129-245.

(Received for publication March 12, 1980; accepted March 31, 1980.) 